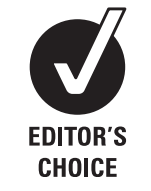

${ }^{1}$ Clinical and Academic Department of Ophthalmology, Great Ormond St Hospital for Children, London, UK ${ }^{2}$ Ilnd Department Ophthalmology, Aristotle University of Thessaloniki, Thessaloniki, Greece ${ }^{3}$ Developmental Biology Unit, UCL Institute of Child Health, London, UK

${ }^{4}$ UPMC Childrens Hospital of Pittsburgh and Eye Center Pittsburgh PA

\section{Correspondence to}

Dr Ken K Nischal, UPMC Childrens Hospital of Pittsburgh and Eye Center, 4401 Penn Avenue, Pittsburgh Pa 15224, USA; nischalkk@upmc.edu

Accepted 25 September 2011 Published Online First 22 October 2011

\title{
The use of punctal plugs in children
}

\author{
A Mataftsi, ${ }^{1}{ }^{2}$ R G Subbu, ${ }^{1}$ S Jones, ${ }^{1}$ K K Nischal ${ }^{1,3,4}$
}

\section{ABSTRACT}

Background/aims To evaluate the safety and efficacy of punctal plugs in children with dry eye syndrome.

Methods A retrospective case series of patients who had an insertion of silicone punctal plugs for dry eye syndrome. Data collected included presenting symptoms, signs, systemic disorders if present, type of anaesthesia and complications by the time of last followup.

Results Twenty-five patients (median age at insertion 7 years, range 1.5-13.8 years) were identified. Median follow-up was 18 months. The commonest symptoms were photophobia, soreness and blepharospasm, and the commonest sign was punctate epithelial erosions. Concurrent systemic disease was present in 18/25 patients. Repeated procedures were carried out in eight of 25 patients. Twenty-four of 35 insertions were performed under general anaesthesia. A substantial improvement in ocular surface disease was noted in all cases: frequency of lubricant use was reduced in eight of 25 and visual acuity improved in 15/25 patients.

Spontaneous extrusion was the commonest complication and occurred within 6 months in 19\% of cases.

Conclusion Dry eye syndrome in children is often accompanied by systemic disease, so in a child with persistent symptoms this should be explored. Punctal plugs offer a safe and effective form of treatment especially as compliance of frequent lubrication is limited in children.

Dry eye can be defined as 'a syndrome in which an unstable tear film inadequately supports the health of the ocular surface epithelium, promotes ocular surface inflammation and stimulates eye pain'. ${ }^{1}$ Causes of dry eye can be divided into tear film abnormalities including aqueous deficiency, mucin deficiency, lipid deficiency and structural abnormalities including epitheliopathy and eyelid incongruence. In adults, this condition is not uncommon, and if left untreated it can be debilitating. Dry eye severe enough to warrant lubrication is rare in children, and compliance of frequent eyedrop instillation is often difficult to achieve in this age group. ${ }^{2}$

First-line treatment strategies are based on lubrication, anti-drainage procedures and antiinflammatory agents, for example, topical ciclosporin. ${ }^{3}$ Although several studies have shown lubrication strategies to be effective they do have disadvantages, such as dependency of symptoms on frequency of instillation, ocular surface damage secondary to preservatives, and inability to replicate the structure and function of the tear film. ${ }^{4}$ The use of punctal plugs has been well documented in adults, and aims to prevent the drainage of natural and artificial tears thus relieving symptoms and signs of the patient. ${ }^{3}$ To the best of our knowledge, there are no previous reports on the use, long-term efficacy and tolerability of punctal plug occlusion in minors. This report summarises our indications and findings following punctal plug use in children and adolescents.

\section{METHODS}

We performed a retrospective case note review of all children from November 1999 to November 2009 presenting with ocular surface disease secondary to structural abnormalities or deficient tear film of any cause who underwent punctal plug occlusion. Cases were ascertained from the clinics of the senior author, by searching the term 'punctal plugs' in all dictated clinical letters. The study was registered with the ethics review board of the UCL, Institute of Child Health and Great Ormond Street Hospital for Children NHS Trust. Patient data collected included presenting symptoms, signs, type and dosage of topical medication, concurrent ocular diagnoses and systemic disorders if present, type of punctual plug used, type of anaesthesia used, modification of signs and symptoms, and any complications by the time of last follow-up.

\section{RESULTS}

Twenty-five children (12 boys, 13 girls) were identified with a median age at time of first punctal plug insertion of 7 years (mean 7.67, SD 3.38, range 1.5-13.8 years). The indication for insertion was based on the presence of ocular surface changes and poor tear film meniscus, with a previous unsuccessful management by lubrication and topical medication alone. Overall, 18 of the 25 patients had a concurrent systemic disorder (table 1).

Signs and symptoms at presentation are summarised in tables 2 and 3 . They all had a degree of corneal staining with fluorescein drops. In two of $25(8 \%)$ cases dry eye was secondary to X-linked hypohydrotic ectodermal dysplasia and keratitis icthyosis deafness, while seven of $25(28 \%)$ patients had aqueous deficient dry eye (triple a syndrome: achalasia, alacrima, hypoadrenalism and Stüve-Wiedemann syndrome). In 14/25 (56\%) there was concurrent meibomian gland dysfunction (posterior margin blepharitis) with presumed evaporative dry eye disease.

Silicone punctal plugs (Altomed, UK) $(0.4 \mathrm{~mm}$, $0.6 \mathrm{~mm}$ or $0.8 \mathrm{~mm}$ sizes) were used in all cases with no peroperative complications. The plugs were placed so that the tip or flat upper flange remained just above the entrance of the punctum; plugs were never buried into the canaliculus. The lower puncta were plugged at an initial stage as they are thought to play a greater role in tear drainage. ${ }^{5}$ Seven 
Table 1 Concurrent systemic diagnoses (no of patients)

\begin{tabular}{ll}
\hline Achalasia, alacrima and hypoadrenalism (AAA) syndrome & 4 \\
Stüve-Wiedemann syndrome & 3 \\
Systemic atopic disease (eczema, asthma) & 2 \\
Developmental delay & 2 \\
Moebius syndrome & 1 \\
X-linked hypohydrotic ectodermal dysplasia & 1 \\
Goldenhar's syndrome & 1 \\
Duane's syndrome & 1 \\
Bilateral microfacial microsomia & 1 \\
Bilateral VI palsy & 1 \\
Sensorineural deafness & 1 \\
Metastatic Ewing's & 1 \\
Transient positive rheumatoid factor & 1 \\
Kearns-Sayre syndrome & 1 \\
Inflammatory linear verrucous epidermal naevus (ILVEN) & 1 \\
Keratitis ichthyosis deafness (KID) syndrome & 1 \\
\hline
\end{tabular}

patients also had plugging of the upper puncta for added effect, at the same (five patients) or a subsequent session (two patients). In five patients, the procedure was repeated when symptoms/signs recurred after spontaneous extrusion of the punctual plug(s). In three patients, a permanent occlusion using thermal cautery was ultimately required for a constant therapeutic effect. A general anaesthetic was necessary in 24/35 procedures when it was deemed necessary by the senior author due to limited cooperation at the slit lamp, with no occurrence of adverse events. In a few cases a second procedure was performed during the same anaesthetic to treat concurrent ocular disease: four patients had lid squeezing for severe meibomian gland dysfunction, one patient had a subconjunctival steroid and antibiotic injection for limbal vernal keratoconjunctivitis, one patient had a lateral tarsorrhaphy for Stüve-Wiedemann syndrome, and one patient had a contact lens put in for corneal dystrophy.

The first follow-up visit occurred at a median of 1 month (mean 1.5, SD 2.07, range 0.1-10 months). The median overall follow-up period after the first punctal plug insertion was 18 months (mean 23.2, SD 19.4, range 0.25-65 months). The three patients in whom the follow-up was shorter than 6 months were under shared care with their local referring eye specialists. Symptomatic improvement was reported in 15 patients who were able to articulate, and attenuation of signs was noted in 24 patients. We also relied clinically on what was reported by parents of younger children (eg, 'she blinks much less now', 'he does not complain of soreness as often as before') but because we did not use a subjective scale we have not included those findings in this study. Visual acuity increased (by one or more line) in 15 patients, and the reported frequency of lubricant use required for symptomatic relief was decreased in eight of the patients.

Table 2 Presenting symptoms (no and percentage of patients)

\begin{tabular}{lc}
\hline Photophobia & $12(48 \%)$ \\
Soreness & $8(32 \%)$ \\
Blepharospasm & $6(24 \%)$ \\
Drop intolerance & $3(12 \%)$ \\
Redness & $2(8 \%)$ \\
Blurry vision & $1(4 \%)$ \\
Watering & $1(4 \%)$ \\
Eye infections & $1(4 \%)$ \\
\hline
\end{tabular}

Table 3 Presenting ocular signs and concurrent ocular diagnoses (no and percentage of patients)

\begin{tabular}{lclc}
\hline Ocular signs & \multicolumn{3}{l}{ Concurrent ocular diagnosis } \\
\hline PEE & $25(100 \%)$ & MGD/BKC & $14(56 \%)$ \\
Corneal scarring & $6(24 \%)$ & Allergic keratoconjunctivitis & $6(24 \%)$ \\
Corneal neovascularisation & $6(24 \%)$ & Absence of meibomian glands & $1(4 \%)$ \\
Anaesthetic corneas & $3(12 \%)$ & Corneal dystrophy (?Meesman's) & $1(4 \%)$ \\
Corneal ulcers & $2(8 \%)$ & Shortening of lids & $1(4 \%)$ \\
Corneal infiltrates & $2(8 \%)$ & & \\
Exposure keratopathy & $2(8 \%)$ & & \\
Filamentary keratitis & $1(4 \%)$ & & \\
Toxic keratopathy & $1(4 \%)$ & & \\
\hline
\end{tabular}

BKC, blepharokeratoconjunctivitis; MGD, meibomian gland dysfunction; PEE, punctate epithelial erosions.

The most common complication was spontaneous extrusion and it occurred within the initial 6 months post-insertion in 19\% of the cases (12/64 plugs). Sixteen (16/64, 21.8\%) plugs fell off the left eye, and $14(14 / 64,25 \%)$ fell off the right eye. Plugs that have remained in situ so far have had a median duration of at least 10.5 months (mean 15.75, SD 16.4, range 0.25-66 months), while those that spontaneously extruded had a median duration of 8 months (mean 10.29, SD 9.9, range 0.25-36 months).

In two patients there was a dubious history of 'eye infection' diagnosed by their general practitioner; however, no evidence of punctal plug-related canalicular infection or conjunctivitis was seen at the immediate subsequent ophthalmic review. In one case, an epithelial breakdown of previously scarred cornea was noted temporally and healed after a few days of prophylactic antibiotic eyedrops with no recurrence. Finally, one patient mentioned a mild foreign body sensation that subsided with time.

\section{DISCUSSION/CONCLUSION}

Dry eye severe enough to cause ocular surface damage in children is rare, with little evidence available for treatment strategies. $^{2} 67$

In our series, a systemic disorder usually coexisted with the dry eye syndrome (18/25 patients, $72 \%)$. In fact, in some of the children it was the ophthalmic examination that prompted a systemic evaluation and lead to the underlying diagnosis. In one other published report of dry eye in children, all 14 children had a concurrent systemic disease. ${ }^{2}$ It is advisable, therefore, for a child presenting with persisting signs or symptoms of dry eye syndrome to have a full paediatric history and clinical examination, so as to reveal or exclude a concurrent systemic condition.

Punctal plugs proved to be an effective treatment for dry eye in our cohort, as they improved signs and/or symptoms of ocular surface disease in all cases. Notably, measurable visual acuity was improved in 15/25 patients, which has also been reported in adults but is of particular importance during ocular development that occurs in childhood. ${ }^{8}$ In seven of 25 children it can be assumed that there was an added therapeutic effect from concurrent procedures undertaken, such as lid squeezing or lateral tarsorrhaphy, but these approaches have different mechanisms of action to punctal plugs and presumably have a combined effect, not replacing one another. In three of 25 children the benefit of punctal occlusion was required on a permanent basis, and in these cases punctal plugs were a necessary step to show the effectiveness and lack of adverse effects of occlusion.

The commonest side-effect in our series was spontaneous extrusion, occurring in 19\% of cases within the first 6 months of 
insertion, and the procedure was repeated if signs or symptoms recurred (eight of 25 patients). The mean duration of spontaneously lost plugs was 10.29 months and that of sustained plugs was 15.75 months. In adults, a reported mean survival time was 19.3 months in a large series of 153 patients. ${ }^{9}$

We cannot confirm that any cases developed an infection that was plug related and none of our cases presented with sideeffects that have been reported in adults such as epiphora, conjunctival erosions, pyogenic granulomas, punctal scarring and canalicular stenosis. ${ }^{9-11}$ In the only child presenting with discomfort, this was mild and transient.

Other concerns with the use of punctal plugs in children have been the necessity of a general anaesthetic for the insertion and possible long-term intolerance. Both of these were not an issue in our series. A short inhalation general anaesthetic without intubation is most of the time sufficient for punctal plug insertion and this may also allow for additional actions, such as a detailed clinical examination, which is limited in children with poor cooperation, as well as an opportunity to perform other minor procedures, as was the case in some of our patients.

On the other hand, the benefits of using an effective therapeutic modality in paediatric dry eye disease are major. The management of dry eye in this age group has particular difficulties: reducing the frequency and increasing the efficacy of lubricants is essential, given the difficulty in putting drops in children's eyes, and maintaining a clear visual axis, as well as minimising blepharospasm and surface irregularities are essential factors in optimising conditions for visual development during the first decade of life.

Other therapeutic approaches are scarce but have been used. ${ }^{2} 6$ One study looked at the management and follow-up of dry eye in 14 children between the ages of 1 and 17 years with the use of artificial tears. ${ }^{2}$ Fourteen per cent developed corneal ulcers and $28 \%$ required conjunctival flap treatment with a mean follow-up time of 4 years. None of our patients, despite having severe systemic conditions such as keratitis icthyosis deafness, developed such complications. An explanation for these differences could be the late presentation to the tertiary centre but also the inefficacy of previous treatment due to the difficulty in putting drops in children's eyes continuously.

The main clinical indication for punctal plug occlusion was previously thought to be aqueous deficient dry eye syndrome. Tai et al ${ }^{9}$ showed a success rate of $76.8 \%$ at 4 weeks after silicone punctal plug insertion for various ocular surface disorders, with a significant reduction in the use of artificial tears. Similarly, punctal plugs were successful in treating a variety of causes of dry eye in our cohort, with more than half of the children presenting with lipid deficiency secondary to meibomian gland dysfunction. As previously reported, this latter feature is often underrecognised in children. ${ }^{12}$

Treatment for dry eye syndrome has historically focused on medical treatment, namely artificial tears, lubricants and antiinflammatory agents. The major shortcoming of this form of treatment in a paediatric population is poor compliance from the patient. Silicone punctal plugs offer a safe, reliable and costeffective alternative and should be considered for use in children with ocular surface disease. The age and persona of the child must be taken into account when deciding the method of anaesthesia for the procedure. In any case when the dry eye syndrome is severe enough to cause ocular surface disease in a child, a systemic cause should be looked for and the ocular microenvironment, especially the lid margins, thoroughly examined.

Competing interests None.

Ethics approval Ethics approval was provided by Great Ormond Street Hospital.

Contributors All authors contributed to the design, data collection and writing of the paper.

Provenance and peer review Not commissioned; externally peer reviewed.

\section{REFERENCES}

1. Murube J. Andrew de Roetth (1893-1981): dacryologist who introduced the term dry eye. Ocul Surf 2004;2:225-7.

2. Mac Cord Medina F, Silvestre de Castro R, Leite SC, et al. Management of dry eye related to systemic diseases in childhood and long term follow-up. Acta Ophthalmol Scand 2007;85:739-44.

3. Calonge M. The treatment of dry eye. Surv Ophthalmol 2001;45(Suppl 2):S227-39.

4. Doughty MJ, Glavin S. Efficacy of different dry eye treatments with artificial tears or ocular lubricants: a systematic review. Ophthalmic Physiol Opt 2009;29:573-83.

5. Carter KD, Nelson CC, Martonyi CL. Size variation of lacrimal punctum in adults. Ophthal Plast Reconstr Surg 1988;4:231-3.

6. Fahnehjelm KT, Törnquist AL, Winiarski J. Dry-eye syndrome after allogeneic stemcell transplantation in children. Acta Ophthalmol 2008;86:253-8.

7. Alves M, Dias AC, Rocha EM. Dry eye in childhood: epidemiological and clinical aspects. Ocul Surf 2008;6:44-51.

8. Goto $\mathbf{E}$, Yagi $Y$, Kaido $\mathrm{M}$, et al. Improved functional visual acuity after punctal occlusion in dry eye patients. Am J Ophthalmol 2003;135:704-5.

9. Tai MC, Cosar CB, Cohen EJ, et al. Improved functional visual acuity after puncta occlusion in dry eye patients. The clinical efficacy of silicone punctal plug therapy. Cornea 2002;21:135-9.

10. Baxter SA, Laibson PR. Punctal plugs in the management of dry eye. Ocul Surf 2004;2:255-65.

11. Boldin I, Klein A, Haller-Schober EM, et al. Long-term follow-up of punctal and proximal canalicular stenoses after silicone punctal plug treatment in dry eye patients. Am J Ophthalmol 2008;146:968-72.e1.

12. Jones SM, Weinstein JM, Cumberland P, et al. Visual outcome and corneal changes in children with chronic blepharokeratoconjunctivitis. Ophthalmology 2007:114:2271-80. 